\title{
Tagung der Schweizerischen Gesellschaft für Geschichte der Medizin und der Naturwissenschaften in Basel
}

\author{
Samstag und Sonntag, den 22./23. September 1956
}

Jedes Jahr im Herbst treten die Naturforscher und Ärzte der Schweiz, die der Schweizerischen Naturforschenden Gesellschaft angehören, zur gemeinsamen Tagung zusammen. Eine ihrer auf 16 Sektionen angewachsenen Fachvereinigungen ist die 12. Sektion für Geschichte der Medizin und der Naturwissenschaften, die unter dem Vorsitz von H. Fischer (Zürich) und W.H.Schopfer (Bern) tagte. Die geschichtlichen Beiträge dieser 136. Jahresversammlung, die zum größten Teil in dem einzigartigen Kollegienhaus der Universität tagte, galten zur Hauptsache der Medizin der Barockzeit in Basel, über die ein Symposium mit der medizinisch-biologischen Sektion durchgeführt wurde.

Diese Unterordnung der Mitteilungen unter ein zentrales Thema hat sich auch dieses Jahr sehr bewährt. Als besonders erfreulich vom Standpunkt der Geschichte einzelner Spezialfächer darf die Tatsache bezeichnet werden, daß die Inhaber der Lehrstühle für Anatomie in Basel und Freiburg (Schweiz) sowie der Vorsteher der Basler ophthalmologischen Klinik ihre Kräfte auch jetzt in den Dienst der Geschichte dieser Disziplinen stellten. Zwei Buchausstellungen, die von Nıкolaus Mani und dem Schreibenden sowie Friedrich Rintelen organisiert wurden, verliehen der Tagung einen besonderen Reiz, indem die Besucher einen Eindruck bekamen von den großartigen Beständen der Basler Universitätsbibliothek. Erfreulich groß war die Zahl der Ärzte aus Basel, die an der Tagung teilnahmen.

Die Samstagsitzung war den freien Mitteilungen gewidmet. Der unermüdliche Hippokrates-Forscher Charles Lichtenthaeler (Leysin) beantwortete die seit der Antike schon oft diskutierte Frage nach der Priorität der Epidemien-Bücher I bis III und des Prognostikon auf Grund medizinischer Argumente dahin, daß die Reihenfolge der Abfassung lauten muß: 3. bis 1. Buch der Epidemien - Prognostikon. - Gerhard Wolf-Heidegger, Vorsteher der Anatomischen Anstalt der Universität Basel, berichtete über eine vom Berner Künstler Albert Anker (1831-1910) in Paris gemalte Fayence des Anatomen Andreas Vesalius (1514 bis 1564), die in den Besitz des genannten Instituts gelangt ist. (Näheres über diesen neuesten Beitrag zur Ikonographie Vesals: siehe Verhandlungen der Naturforschenden Gesellschaft Basel 67 (1956), Nr. 2, 133-40, mit Schwarzweißreproduktion des Porträts.) - Adolf Faller, Ordinarius für Anatomie in Freiburg (Schweiz) untersuchte den «Einfluß des Cartesianismus auf Niels Stensen», einen der hervorragendsten dänischen Ärzte, der seit kurzem im Brennpunkt medizinhistorischer Bemühungen steht. Aus der biographisch-chronologisch aufgebauten Skizze wird deutlich, wie scharf der vielseitige Biologe die Mängel der physiologischen Deduktionen des französischen Denkers und damit auch die 
aus ihnen abgeleiteten philosophischen Folgerungen vor allem zum Leib-SeeleProblem zu kennzeichnen wußte. Eine Reihe von Diapositiven diente als wertvolle Illustration des Ausgeführten. - Als emeritierter Zoologe behandelte Hubert Erhard (Adelholzen, Oberbayern) «Paracelsus, die Bienen und die Signaturen ", wobei deutlich wurde, wie Hohenheim als einer der ersten in der Naturbetrachtung vom anthropozentrischen Standpunkt abrückte (Beispiel: Bienen und Blütenfarbe). - Den Schluß der Sitzung bildete ein teils historischer, teils hämatologischer Exkurs von E. Undritz (Basel) über «Die larvierte Anämie von Sahli», dessen 100. Geburtstag (vgl. unten) Anlaß bot zu dieser interessanten Darstellung eines speziellen Problems, das die weitblickende Forschung des Berner Klinikers aufzeigte.

In der Geschäftssitzung vom Sonntagmorgen orientierte der Präsident über den Kongreß für die Geschichte der Wissenschaften in Florenz und die neuesten Entwicklungen organisatorischer Natur. Sodann gedachte H. Fischer der beiden verstorbenen Mitglieder der Gesellschaft: Prof. Dr. Bernhard Milt in Zürich (1896-1956), des Inhabers des ersten Lehrstuhls für Geschichte der Medizin in der Schweiz (vgl. den Nekrolog in Heft 1/2, 1956, dieser Zeitschrift), und Prof. Dr. Louis Michaud (geb. 8. Juni 1880 in Bern, gest. 9. Juli 1956 in Lausanne), emeritierten Direktors der Medizinischen Klinik in Lausanne, der seit 1922 der Gesellschaft angehört hatte und namentlich auch im Pura-Kreis eine Lücke hinterlassen wird. Auf die vorgesehene Würdigung seines Lehrers Hermann Sahli durch Michaud mußte die Gesellschaft leider verzichten.

Die vier Vorträge zur «Medizin der Barockzeit in Basel» ergänzten sich in fruchtbringender Weise. Über ihre Vorbereitung und Anfänge sprach N.MANI (Basel), der im wesentlichen «Basel als Zentrum der medizinisch-humanistischen Bewegung » skizzierte, wobei der Vortragende, anschließend an Vorarbeiten (vgl. diese Zeitschrift 10 (1956) 29-52) die verschiedenen Humanistenärzte einheimischer und fremder Provenienz sowie die wichtigsten Drucker insbesondere der Erstausgaben vor Augen führte. - Thematisch schloß sich J. Karchers (Basel) fein gezeichnete Charakteristik von «Thomas Erastus (1524-1583), dem unversöhnlichen Gegner des Paracelsus» an, die einen wertvollen Beitrag zur Medizin des konfessionellen Zeitalters in Heidelberg und Basel brachte. - Nach diesen vorbereitenden Referaten wurde von H. Buess (Basel) «Physiologie und Pathologie in Basel zur Zeit des Barocks» erörtert, wobei zunächst eine zeitliche Abgrenzung, dann eine allgemeine Kennzeichnung des Barocks in Deutschland und Basel versucht wurden, bevor zwei Teilgebiete aus dem Lebenswerk der bedeutendsten Basler Ärzte im Zeitraum von etwa 1670-1724 kurz zur Sprache kamen. - F. Rintelen, Vorsteher der Augenheilanstalt, schloß in anregender Weise die Tagung mit seinem fachgeschichtlichen Referat «Die Ophthalmologie in Basel zur Zeit des Barocks », in dem einleitend in Medizin und Naturwissenschaften auf die Unterschiede zwischen Deutschland und Frankreich und auf 
die Stellung Basels in jener bewegten Zeit hingewiesen wird. Ein Überblick über die an wertvollen Ansätzen so wichtige physiologische Optik (Kepler, Scheiner, Descartes) leitete über zur Schilderung der wissenschaftlichen Leistungen und der praktischen Seite der Augenheilkunde in Basel. Die Referate zum Haupthema werden voraussichtlich in dieser Zeitschrift zur Veröffentlichung gelangen.

Dank dem Entgegenkommen der Leitung der Universitätsbibliothek, der Anatomischen Anstalt und des Staatsarchivs konnten zwei Ausstellungen von Büchern, Porträts und Manuskripten gezeigt werden, die den medizinischen $\mathrm{Hu}$ manismus sowie die Medizin und die Ophthalmologie in Basel zur Zeit des Barocks betrafen und lebhaftes Interesse fanden, nachdem auch in einer vorgängigen Presseorientierung darauf aufmerksam gemacht worden war. N.MANI, F. Rintelen und H.Buess teilten sich in die Führungen.

Den feierlichen Abschluß brachte der Vortrag von W. Frey (Oberhofen BE) «Zur Erinnerung an Hermann Sahli», den so bedeutenden Kliniker der Berner Hochschule (geb. 23. Mai 1856, gest. 28. April 1933), dessen Verdienste besonders um den Ausbau der Magendiagnostik, der Hämatologie, der Tuberkuloseforschung, der Hämodynamik und der Neurosenlehre in helles Licht gerückt wurden. Beim Mittagessen im Kreise der Sektion und bei den Veranstaltungen des gesamten Kongresses wurden manche wertvollen Anregungen ausgetauscht und neue Aspekte der Geschichtswissenschaft in der Medizin diskutiert.

H. Buess (Basel) 